\title{
Acute pulmonary histoplasmosis masquerading as miliary tuberculosis in a non-endemic region
}

\author{
Mehul Agarwal ${ }^{\circ}$, Benhur Joel Shadrach \\ Department of Pulmonary Medicine, All India Institute of Medical Sciences, Jodhpur, India
}

A 28-year-old female who worked as a house maid presented with fever, troublesome cough, progressive breathlessness, and anorexia which had been present for 25 days. She had tachypnea, tachycardia, and a falling peripheral oxygen saturation. Bilateral inspiratory crepitations were heard on chest auscultation. An arterial blood gas measurement showed hypoxemic respiratory failure. She was in close contact with an active case of pulmonary tuberculosis. A sputum test for acid fast bacilli (AFB) and GeneXpert were negative for tuberculosis. Chest radiograph revealed bilateral nodular opacities. High-resolution computerized tomography (HRCT) of the thorax disclosed bilateral pulmonary nodules in a random distribution (miliary pattern) as well as mediastinal lymphadenopathy (Figure 1A). She was diagnosed clinically with pulmonary tuberculosis and initiated on anti-tubercular treatment (ATT). However, her symptoms worsened and ATT was discontinued. There was a palpable right supraclavicular lymph node and an excision biopsy revealed granulomatous inflammation (Figure 1B). Ziehl-Neelsen stain stain for AFB and GeneXpert were negative. Fungal staining revealed Histoplasma capsulatum (Figure 1C). She was diagnosed with acute pulmonary histoplasmosis and initiated on Amphotericin-B liposomal injection $4 \mathrm{mg} / \mathrm{kg} /$ day intravenously for 14 days followed by oral Itraconazole $200 \mathrm{mg}$ twice daily. There was a significant clinico-radiological response to the antifungal therapy.

Histoplasma capsulatum is a dimorphic fungus which grows as a mould in soil (infective form) and as yeast in the host's tissue (pathogenic form) [1]. Two varieties of histoplasma are known to infect humans H. capsulatum var. capsulatum and $H$. capsulatum var. duboisii. It is endemic in Central and North America but sporadic cases have been reported in eastern India along the Gangetic plains. It can involve almost any organ system but pulmonary and mediastinal involvement are most common [2]. Acute pulmonary histoplasmosis may vary in its clinical presentation. It may present as an asymptomatic illness or as a severe disease progressing to acute respiratory failure. Clinical features include fever, malaise, breathlessness, dry cough,
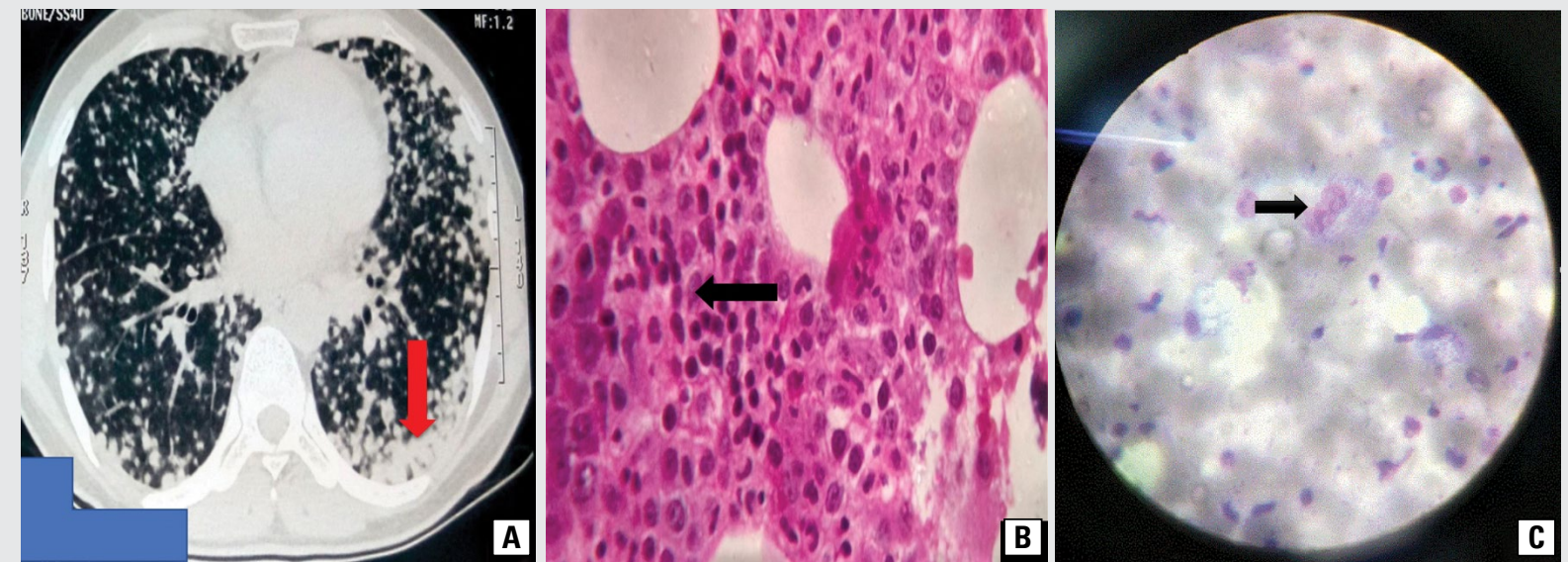

Figure 1. A. HRCT thorax axial reformatted images at the level of inferior pulmonary vein showing bilateral nodules in random distribution with conglomeration of nodules seen at lung bases (red arrow); B. Lymph node biopsy sections H\&E stain $400 \times$ magnification showing multiple epithelioid granuloma (black arrow); C. Lymph node biopsy section Giemsa stain 1000x magnification showing capsulated yeast form of histoplasma (black arrow)

Address for correspondence: Benhur Joel Shadrach, Department of Pulmonary Medicine, All India Institute of Medical Sciences, Jodhpur, India; e-mail: benjoe6326@gmail.com Conflict of interest: None declared

DOI: 10.5603/ARM.a2021.0051 | Received: 26.08.2020 | Copyright (C 2021 PTChP | ISSN 2451-4934 | e-ISSN 2543-6031

This article is available in open access under Creative Common Attribution-Non-Commercial-No Derivatives 4.0 International (CC BY-NC-ND 4.0) license, allowing to download articles and share them with others as long as they credit the authors and the publisher, but without permission to change them in any way or use them commercially. 
and chest pain. Chest imaging usually reveals nodular opacities mimicking miliary shadows and mediastinal adenopathy. Although miliary opacities on chest imaging have a long list of differentials, patients are frequently diagnosed as having either tuberculosis (in the developing world) or sarcoidosis (in the developed world) and are initiated on empirical therapy [3]. Since, the symptomatic and severe form of acute pulmonary histoplasmosis can be fatal, early and definitive diagnosis is imperative. The best method is via direct detection of the organism by histopathology or cytology. Other diagnostic options include antigen detection, culture, serology, skin prick tests, and molecular tests [4]. Treatment is not required in asymptomatic or mild cases. Severe cases are treated with liposomal Amphotericin B (3-5mg/kg/day) or deoxycholate Amp B (0.7-1 mg/ $\mathrm{kg} /$ day) intravenously for 7-14 days followed by oral Itraconazole (200 mg thrice a day for 3 days followed by twice daily) for a total of 6 weeks [5].

Histoplasmosis is not uncommon in non-endemic regions but is an under-recognized entity because the clinico-radiological and pathological profile mimics tuberculosis, the most common granulomatous disease in this part of the world. Besides, the environmental cause of fungal infections in non-endemic regions is yet to be explored leading to most cases being misdiagnosed. Hence, a high clinical suspicion and awareness is required for its diagnosis.

\section{References:}

1. Evrard S, Caprasse P, Gavage P, et al. Disseminated histoplasmosis: case report and review of the literature. Acta Clin Belg. 2018; 73(5): 356-363, doi: 10.1080/17843286.2017.1376454, indexed in Pubmed: 28954600.

2. Kauffman C. Pulmonary histoplasmosis. Current Infectious Disease Reports. 2001; 3(3): 279-285, doi: 10.1007/s11908-001-0031-6.

3. Furqan M, Butler J. Miliary pattern on chest radiography: TB or not TB? Mayo Clin Proc. 2010; 85(2): 108, doi: 10.4065/ mcp.2009.0523, indexed in Pubmed: 20118384.

4. Wheat LJ. Approach to the diagnosis of the endemic mycosis. Clin Chest Med. 2009; 30: 379-89.

5. Wheat LJ, Freifeld AG, Kleiman MB, et al. Clinical practice guidelines for the management of patients with histoplasmosis: 2007 update by the Infectious Diseases Society of America. Clin Infect Dis. 2007; 45(7): 807-825, doi: 10.1086/521259, indexed in Pubmed: 17806045 . 